\title{
Beta-Blocker Type Effect on Substrate Oxidation during HIIE in Heart Failure Patients: Pilot Data
}

\author{
Paula Aver Bretanha Ribeiro, ${ }^{1,2,3}$ Eve Normandin, ${ }^{1,2,4}$ Philippe Meyer, ${ }^{5}$ Martin Juneau, ${ }^{1,2}$ Michel White, ${ }^{1,2}$ \\ Anil Nigam, 1,2 Mathieu Gayda ${ }^{1,2}$ \\ Cardiovascular Prevention and Rehabilitation Centre (ÉPIC), Montreal Heart Institute, ${ }^{1}$ Montreal, Quebec - Canada \\ Department of Medicine, Faculty of Medicine, University of Montreal, ${ }^{2}$ Montreal, Quebec - Canada \\ Hospital de Clínicas de Porto Alegre, Universidade Federal do Rio Grande do Sul (UFRS), ${ }^{3}$ Porto Alegre, RS - Brazil \\ Faculty of Physical Education and Sports, University of Sherbrooke, ${ }^{4}$ Sherbrooke, Quebec - Canada \\ Division of Cardiology, University Hospital, Faculty of Medicine, University of Geneva, ${ }^{5}$ Geneva - Switzerland
}

\begin{abstract}
The effect of third and second-generation type of betablocker on substrate oxidation especially during high-intensity exercises are scarce.

The objective of the study is to explore differences of beta-blocker regimens (vasodilating vs. non-vasodilating beta-blockers) for substrate oxidation during in high-intensity intermittent exercise (HIIE) in chronic heart failure and reduced ejection fraction ( $\mathrm{HFrEF})$.
\end{abstract}

Eighteen CHF males (58.8 \pm 9 years), 8 under use of $\beta 1$ specific beta-blockers+alfa 1-blocker and 10 using $\beta 1$ non-specific beta-blockers, were randomly assigned to 4 different HIIE, in a cross-over design. The 4 protocols were: 30 seconds ( $A$ and $B$ ) or 90 seconds (C and D) at 100\% peak power output, with passive (A and C) or active recovery (50\% of $\mathrm{PPO}$; $\mathrm{B}$ and D). Energy expenditure (EE; $\mathrm{kcal} / \mathrm{min})$, quantitative carbohydrate $(\mathrm{CHO})$ and lipid oxidation $(\mathrm{g} / \mathrm{min})$ and qualitative (\%) contribution were calculated. Two-way ANOVA and Bonferroni post-hoc test were used ( $p$-value $\leq 0.05$ ) to compare $\mathrm{CHO}$ and lipid oxidation at rest and at $10 \mathrm{~min}$.

Total exercise time or EE did not show differences for beta-blocker use. The type of beta-blocker use showed impact in $\mathrm{CHO}(\%)$ and lipid ( $\mathrm{g} / \mathrm{min}$ and \%) for rest and $10 \mathrm{~min}$, but absolute contribution of $\mathrm{CHO}(\mathrm{g} / \mathrm{min})$ was different just at $10 \mathrm{~min}$ (Interaction $\mathrm{p}=0.029$ ). Higher $\mathrm{CHO}$ oxidation was found in vasodilating beta-blockers when comparing to non-vasodilating.

According to our pilot data, there is an effect of beta-blocker type on substrate oxidation during HIIE, but no influence on EE or exercise total time in HFrEF patients.

\section{Introduction}

To reduce sympathetic nervous system activation and improve myocardium contractility, morbidity and mortality,

\section{Keywords}

Heart Failure, Adrenergic beta-Antagonists; Stroke Volume, Chemical Oxidation, Exercise.

Mailing Address: Paula Aver Bretanha Ribeiro •

Cardiovascular Prevention and Rehabilitation Centre (ÉPIC), 5055 St Zotique

East, Montreal, Quebec, H1T1N6, Canada

E-mail: paulaabribeiro@gmail.com

Manuscript received January 27, 2018, revised manuscript April 26, 2018, accepted August 15, 2018

DOI: 10.5935/abc.20190039 most of the chronic heart failure (CHF) patients are under beta-blocker use. ${ }^{1}$ Besides all benefits, there is some evidence showing reduced glycemic control, weight gain, insulin secretion inhibition and resistance and dyslipidemia in resting states in patients under beta-blocker regimen. ${ }^{2,3}$ Also, it was showed that the use of beta blockers could remodel substrate use during moderate-intensity continuous exercise. ${ }^{4}$

The third generation of beta-blockers, described as vasodilating $\beta$ - blockers (i.e. $\beta$ non-selective $+\alpha$-1 blocker), seem to have a beneficial effect comparing to previous ones ( $\beta$-selective). Because of its lack of effect on $\alpha 1$ - adrenergic receptors, non-vasodilating beta blockers can induce vasoconstriction, reducing blood flow and glucose uptake at muscular level. ${ }^{2,3}$ But when comparisons between carvedilol vs. metoprolol were made concerning NYHA no changes were found. ${ }^{5}$ However, there is a lack of evidence of comparison of the type of beta-blocker during exercise, especially during high-intensity exercises (i. e. vasodilating vs. non-vasodilating).

Regarding exercise intensity, there is an increasing use of high-intensity interval training programs in cardiac rehabilitation sites. Enough evidence is provided about the superior benefits of this modality for maximal oxygen uptake $\left(\mathrm{VO}_{2}\right.$ max) and quality of life when compared to moderate continuous training. ${ }^{6}$ However, still a lack of evidence about different high-intensity interval exercise (HIIE) prescriptions in terms of optimal intensity, duration of bouts, recovery time and type (passive or active). ${ }^{7}$ Also, not enough evidence about substrate utilization in cardiac rehabilitation context, especially what concerns to medication use. ${ }^{4,8}$ We believe that vasodilating beta-blockers could especially influence carbohydrate $(\mathrm{CHO})$ oxidation, and this may reflect in total time of exercise session.

Therefore, for this study, we compared substrate oxidation, energy expenditure (EE) and total time of HIIE protocols performed by $\mathrm{CHF}$ with reduced left ejection fraction (HFrEF), under two different beta-blocker regimens (vasodilating vs. non-vasodilating).

\section{Methods}

\section{Participants}

Twenty stable HFrEF were recruited from the heart failure ambulatory at the Montreal Heart Institute. This is a sub-study, inclusion criteria and exclusion criteria have been detailed in the previous publication, ${ }^{7}$ and clinical information was 


\section{Brief Communication}

obtained from medical records. The study was performed in a cross-over design, all patients participated in the same exercise sessions in random order (generated by randomizer.org), and every single session being separated by one week ( 5 weeks for complete protocol). No changes in medication were made during the evaluations. The protocol was accepted by the Ethics Committee of the Montreal Heart Institute (08-1023), all procedures in agreement with Helsinki declaration and written informed consent was obtained from all patients.

\section{Maximal cardiopulmonary exercise test}

Maximal cardiopulmonary exercise testing was performed according to previously published methodology. ${ }^{7-9}$ In short, the maximal exercise protocol was performed on a cycle ergometer (Ergoline 800S, Bitz, Germany), speed was settled at 60 RPM, and the power was increased by $10 \mathrm{~W}$ every minute until exhaustion. Gas exchanges variables were measured breath by breath during testing and then averaged every $15 \mathrm{~s}$. Peak power output (PPO) was defined as the power output reached the last fully completed stage. Electrocardiographic activity was monitored continuously using an 8-lead ECG (Marquette, Missouri, USA).

\section{HIIE sessions}

The exercise sessions were based on previously published methodology in patients with HFrEF.? HIIE protocols were all prescribed at $100 \%$ PPO, based on CPET and differed in interval duration (30 seconds for protocols A and B vs 90 seconds for protocols $C$ and D) and type of recovery (active recovery at $50 \%$ of PPO for protocols $\mathrm{B}$ and $\mathrm{D}$ vs passive recovery [0\% of $\mathrm{PPO}]$ for protocols $\mathrm{A}$ and $\mathrm{C}$ ). The exercise time and recovery were designed as 1:1 ratio. Each patient exercised for a maximal time of 30 min or until exhaustion due to fatigue, dyspnea, dizziness, or inability to maintain pedal cadence at $60 \mathrm{rpm}^{7}$

\section{Substrate oxidation and energy expenditure calculation}

EE was calculated using Weir equation. ${ }^{10}$ Substrate oxidation ( $\mathrm{CHO}$ and lipid) was calculated from gas exchange using the Frayn equation ${ }^{11}$ (in $\mathrm{g} / \mathrm{min}$ ) and with a respiratory exchange ratio values (in \%) using a table of non-protein respiratory quotient. ${ }^{12}$

\section{Statistical analysis}

Results are expressed as mean \pm SD for clinical characteristics and described as $\mathrm{n}(\%)$ for beta blocker regimen (Table 1). This is an exploratory analysis from our previous studies, $^{7-9}$ all HIIE sessions that lasted more than 10 min of exercise were included. The substrates were compared in two time-points: rest and end of $10 \mathrm{~min}$, both averages of $3 \mathrm{~min}$ measurements. EE (Kcal/min; EE), $\mathrm{CHO}$ and lipid oxidation, in $\mathrm{g} / \mathrm{min}$ and total contribution (\%), were compared during HIIE protocols using a two-way ANOVA for beta-blocker type and time factors. The Bonferroni post-hoc test with a $p$ value $\leq 0.05$ was used. Student`s t-test was used to compare the exercise total time between types of beta-blocker. All analyses were performed using IBM SPSS Statistics software, version 21 and Statview.

\section{Results}

The patients' characteristics are described in Table 1, two patients were excluded for this analysis because did not achieve the minimum of 10 min exercise. Both groups were similar for clinical characteristics, except for systolic and diastolic blood pressures. Total exercise time was not different between the beta-blockers types (non-vasodilating group $=1377 \pm 505 \mathrm{~s}$; vasodilating group $=1371 \pm 503 \mathrm{~s}$; $p=0.962$ ). Also, no differences were found for $E E$ for group or interaction (group $p=0.203$; time $p<000.1$ and interaction $\mathrm{p}=0.867)$. Differences in $\mathrm{CHO}\left(\mathrm{mg} / \mathrm{min}^{*} \mathrm{Kg}^{-1}\right.$ and $\% ; p=0.012$ and $p=0.0006)$ and lipids $\left(\mathrm{mg} / \mathrm{min}^{*} \mathrm{Kg}^{-1}\right.$ and $\% ; p=0.0017$ and $p=0.0083$ ) were found for group and time analysis, and interaction was found just for $\mathrm{CHO}$ $\left(\mathrm{mg} / \mathrm{min}^{*} \mathrm{Kg}^{-1} ; \mathrm{p}=0.03\right)$ (Figure 1$)$.

\section{Discussion}

Our results showed a different effect on substrate oxidation depending on the type of beta-blocker generation. We believe that the decision of beta-blocker use can benefit the substrate oxidation during exercise and can be chosen accordingly with patients' necessity. Because of their effect on alfa1 - adrenergic receptors, vasodilating beta-blockers like carvedilol could benefit $\mathrm{CHO}$ oxidation, as shown in our results for absolute and relative values, comparing to non-vasodilation beta-blockers. Because these blocking agents can have a different effect on the circulatory and respiratory systems, previously they were known to potentially reduce exercise capacity in heart failure patients. ${ }^{13}$ Recent evidence has shown some improvement, with a different combination of drugs, vasodilating beta-blockers demonstrated positive effect increasing insulin sensitivity comparing to non-vasodilating ones ${ }^{14}$ and probably remodelling substrate oxidation.

According to literature, HIIE requires greater energetic demand from the muscular system, ${ }^{15}$ and therefore should be accompanied by higher $\mathrm{CHO}$ oxidation. The increasing use of HIIE in a clinical context is due to its superiority compared to continuous exercise training to improve $\mathrm{VO}_{2 \text { peak' }}$ with similar effects on left ventricular function, safety and exercise compliance. ${ }^{6,16}$ In our previous work, ${ }^{9}$ we showed substrate oxidation differences between HIIE protocols, and the individual variances lead us to explore the potential muscle metabolism differences that could be related to betablocker use. Also, there is still little data available on substrate oxidation and the effect of pharmacological agents in heart failure patients, ${ }^{3}$ especially during high-intensity exercise, so we believe we are providing interesting initial data to raise interest on the subject.

The major limitation of our study is the sample size, but we believe appropriate for a pilot study to encourage further investigation. Also, because of the lack of a placebo group, we cannot investigate the actual effect of beta-blocker on substrate oxidation, but differences between different regimens. Since more than $90 \%$ of our HFrEF patients are under some beta-block medication, we did not consider the possibility to suspend or change patients' medication. 
Brief Communication

Table 1 - Baseline Clinical Characteristics according to the type of beta-blocker class

\begin{tabular}{|c|c|c|}
\hline \multirow{2}{*}{ Clinical Variables } & \multicolumn{2}{|c|}{ Beta-blocker class } \\
\hline & Non-vasodilating $n=10$ & Vasodilating $n=8$ \\
\hline Age (years) & $59.3 \pm 9.8$ & $58.0 \pm 8.5$ \\
\hline $\mathrm{BMl}\left(\mathrm{kg} / \mathrm{m}^{2}\right)$ & $30.0 \pm 4.0$ & $28.0 \pm 3.7$ \\
\hline $\operatorname{LVEF}(\%)$ & $29 \pm 7$ & $27 \pm 6$ \\
\hline $\mathrm{SBD}(\mathrm{mmHg})$ & $129 \pm 20$ & $108 \pm 17^{*}$ \\
\hline $\mathrm{DBP}(\mathrm{mmHg})$ & $75 \pm 10$ & $61 \pm 15^{\star}$ \\
\hline \multicolumn{3}{|l|}{ NYHA functional class } \\
\hline । & $1(10 \%)$ & $4(50 \%)$ \\
\hline$\|$ & $9(90 \%)$ & $3(37.5 \%)$ \\
\hline III & 0 & $1(12.5 \%)$ \\
\hline \multicolumn{3}{|l|}{ Etiology of heart failure } \\
\hline Ischemic heart disease & $6(60 \%)$ & $4(50 \%)$ \\
\hline Idiopathic dilated cardiomyopathy & $4(40 \%)$ & $4(50 \%)$ \\
\hline \multicolumn{3}{|l|}{ Medical history } \\
\hline Diabetes mellitus & $1(10 \%)$ & $3(37.5 \%)$ \\
\hline Hypertension & $6(60 \%)$ & $4(50 \%)$ \\
\hline \multicolumn{3}{|l|}{ Medications } \\
\hline ACE inhibitors or ARBs & $10(100 \%)$ & $8(100 \%)$ \\
\hline Digoxin & $2(20 \%)$ & $4(50 \%)$ \\
\hline Furosemide & $8(80 \%)$ & $6(75 \%)$ \\
\hline Spironolactone & $4(40 \%)$ & $4(50 \%)$ \\
\hline \multicolumn{3}{|l|}{ Devices } \\
\hline ICD & $7(70 \%)$ & $6(75 \%)$ \\
\hline CRT & $1(10 \%)$ & $3(37.5 \%)$ \\
\hline \multicolumn{3}{|l|}{ Maximal exercise variables } \\
\hline Peak power output (Watts) & $108 \pm 33$ & $110 \pm 31$ \\
\hline $\mathrm{VO}_{2 \text { peak }}(\mathrm{L} / \mathrm{min})$ & $1598 \pm 507$ & $1478 \pm 422$ \\
\hline $\mathrm{VO}_{2 \text { peak }}(\%$ predicted $)$ & $63 \pm 13$ & $59 \pm 12$ \\
\hline $\mathrm{VO}_{2 \text { peak }}(\mathrm{mL} / \mathrm{min} / \mathrm{kg})$ & $17.3 \pm 4.6$ & $18.3 \pm 4.6$ \\
\hline
\end{tabular}

Values are presented as means \pm SDs, or numbers of patients (percentages). BMI: body mass index; LVEF: left ventricle ejection fraction; SBP: systolic blood pressure; DBP: diastolic blood pressure; ACE: angiotensin-converting enzyme; ARBs: angiotensin II receptor blockers; ICD: implantable cardioverter-defibrillator; CRT: cardiac resynchronization therapy; $\mathrm{VO}_{2}$ : oxygen uptake; $\mathrm{VO}_{2 \text { peak }}$ : peak oxygen uptake. ${ }^{*} p<0.05$.

\section{Conclusion}

In short, according to our pilot data, carvedilol seems to facilitate $\mathrm{CHO}$ oxidation during HIIE and should be considered when possible, for patients under high-intensity exercise programs. However, how the use of different beta-blockers agents (ex: vasodilating) could impact muscle metabolism during various acute and chronic exercise training programs in these patients need to be better explored.

\section{Acknowledgement}

The authors are grateful to ÉPIC Foundation, Montreal Heart Institute Foundation and Conselho Nacional de Desenvolvimento Científico e Tecnológico (CNPq- Brazil) for grant support.

\section{Author contributions}

Conception and design of the research: Ribeiro PAB, Juneau $M$, White M, Nigam A, Gayda M; acquisition of data: Normandin E, Meyer P; analysis and interpretation of the data: Ribeiro PAB, Normandin E, Meyer P, White M, Nigam A, Gayda M; statistical analysis: Ribeiro PAB; obtaining funding: Ribeiro $P A B$, Juneau $M$, Gayda M; writing of the manuscript: Ribeiro PAB, Gayda M; critical revision of the manuscript for intellectual content: Ribeiro $\mathrm{PAB}$, Normandin E, Meyer P, Juneau M, White M, Nigam A, Gayda M.

\section{Potential Conflict of Interest}

No potential conflict of interest relevant to this article was reported. 


\section{Brief Communication}

A

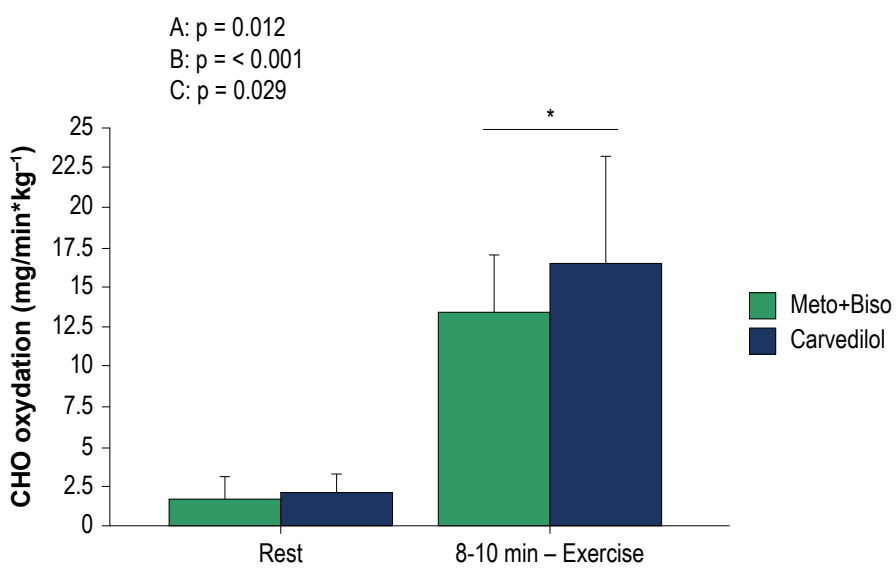

B

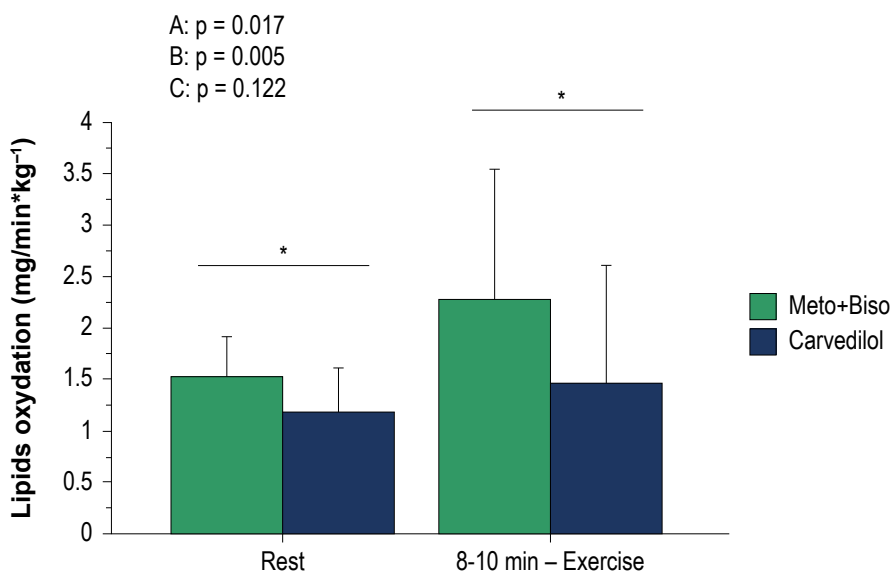

Figure 1 - A) Carbohydrate oxidation by group at rest and 8-10 minutes high-intensity interval exercise. B) Lipids oxidation by groups at rest and 8-10 minutes highintensity interval exercise. ${ }^{*} p<0.05$ for groups; ANOVA $p$ value results: A: group; B: time; C: interaction.

\section{Sources of Funding}

This study was funded by the ÉPIC Fondation, the Montreal Heart Institute Foundation and the CNPq.

\section{Study Association}

This article is part of the Post-Doctoral fellowship submitted by Paula Aver Bretanha Ribeiro, from University of Montreal. 


\section{References}

1. Naegele M, Flammer AJ, Enseleit F, Ruschitzka F. Medical therapy of heart failure with reduced ejection fraction: current evidence and new developments. Swiss Med Wkly. 2016 Mar;146:w14295.

2. Ladage D, Schwinger RH, Brixius K. Cardio-selective beta-blocker: pharmacological evidence and their influence on exercise capacity. Cardiovasc Ther. 2013;31(2):76-83.

3. Fonseca VA. Effects of beta-blockers on glucose and lipid metabolism. Curr Med Res Opin. 2010;26(3):615-29.

4. Smith J, Ferland A, Methot J, Brassard P, Lacroix S, Poirier P, et al. The beta-1 adrenergic antagonist, atenolol, decreases acylation stimulating protein, exercise capacity and plasma free fatty acids in men with type 2 diabetes. Nutr Metab Cardiovasc Dis. 2012;22(6):495-502.

5. Blanchet M, Ducharme A, Racine N, Rouleau JL, Tardif JC, Juneau M, et al. Effects of cold exposure on submaximal exercise performance and adrenergic activation in patients with congestive heart failure and the effects of beta-adrenergic blockade (carvedilol or metoprolol). Am J Cardiol. 2003;92(5):548-53.

6. Ismail H, McFarlane JR, Nojoumian AH, Dieberg G, Smart NA. Clinical outcomes and cardiovascular responses to different exercise training intensities in patients with heart failure: a systematic review and metaanalysis. JACC Heart Fail. 2013;1(6):514-22

7. Meyer P, Normandin E, Gayda M, Billon G, Guiraud T, Bosquet L, et al. Highintensity interval exercise in chronic heart failure: protocol optimization. J Card Fail. 2012;18(2):126-33.

8. Kraal JJ, Sartor F, Papini G, Stut W, Peek N, Kemps HM, et al. Energy expenditure estimation in beta-blocker-medicated cardiac patients by combining heart rate and body movement data. Eur Prev Cardiol. 2016;23(16):1734-42.

9. Ribeiro PA, Normandin E, Meyer P, Juneau M, Nigam A, Gayda M Comparison of carbohydrate and lipid oxidation during different highintensity interval exercise in patients with chronic heart failure. Am J Phys Med Rehabil. 2017;96(1):50-4.

10. Weir JB. New methods for calculating metabolic rate with special reference to protein metabolism. 1949. Nutrition. 1990;6(3):213-21.

11. Frayn KN. Calculation of substrate oxidation rates in vivo from gaseous exchange. J Appl Physiol Respir Environ Exerc Physiol. 1983;55(2):628-34.

12. Peronnet $F$, Massicotte $D$. Table of nonprotein respiratory quotient: an update. Can J Sport Sci 1991;16(1):23-9.

13. Beauvais F. [Physical training with beta-blockers in chronic heart failure]. Ann Cardiol Angeiol (Paris). 2010;59(4):229-33.

14. Jacob S, Rett K, Wicklmayr M, Agrawal B, Augustin HJ, Dietze GJ. Differential effect of chronic treatment with two beta-blocking agents on insulin sensitivity: the carvedilol-metoprolol study. J Hypertens. 1996;14(4):489-94.

15. Gayda M, Normandin E, Meyer P, Juneau M, Nigam A. Comparison of carbohydrate and lipid oxidation during continuous and intermittent exercise in patients with chronic heart failure. Can J Cardiol. 2013;29(8):990-2.

16. Haykowsky MJ, Timmons MP, Kruger C, McNeely M, Taylor DA, Clark AM Meta-analysis of aerobic interval training on exercise capacity and systolic function in patients with heart failure and reduced ejection fractions. Am J Cardiol. 2013;111(10):1466-9. 